\title{
High-frequency ultrasound array element using thermoelastic expansion in an elastomeric film
}

\author{
T. Buma \\ Applied Physics Program, University of Michigan, Ann Arbor, Michigan 48109-2125 \\ M. Spisar \\ Department of Biomedical Engineering, University of Michigan, Ann Arbor, Michigan 48109-2125 \\ M. O'Donnella) \\ Applied Physics Program and Department of Biomedical Engineering, University of Michigan, Ann Arbor, \\ Michigan 48109-2125
}

(Received 11 May 2001; accepted for publication 31 May 2001)

\begin{abstract}
The thermoelastic effect was used to produce high-frequency, broadband ultrasound in water. A pulsed diode laser, followed by an erbium-doped fiber amplifier, was focused onto a light-absorbing film deposited on a glass substrate. Conversion efficiency was improved by over $20 \mathrm{~dB}$ using an elastomeric film instead of a more commonly used metallic one. Radiation pattern measurements show that considerable energy is radiated at $+1-45^{\circ}$ for frequencies beyond $50 \mathrm{MHz}$. These results show that the thermoelastic effect can be used to produce phased arrays for high-frequency ultrasound imaging. (C) 2001 American Institute of Physics. [DOI: 10.1063/1.1388027]
\end{abstract}

Optoacoustics is a promising alternative to piezoelectricity for high-frequency arrays. The size and location of a focused laser beam defines an array element. Optical generation of ultrasound is possible through a variety of mechanisms, the most common being the thermoelastic effect. ${ }^{1}$ A major drawback to thermoelastic generation has been poor conversion efficiency. It can be improved, however, by choosing a material possessing a high thermal expansion coefficient, $\alpha$, for the light-absorbing structure. ${ }^{2,3}$ Elastomeric materials are particularly attractive since they are often biologically safe, have large $\alpha$, and can be deposited in thin films by spin casting. ${ }^{4}$ In particular, polydimethylsiloxane $(\mathrm{PDMS})^{5}$ has a bulk linear coefficient of thermal expansion of $\alpha_{L}=310 \times 10^{-6} \mu \mathrm{m} / \mu \mathrm{m} /{ }^{\circ} \mathrm{C}$. This is almost five times higher than water and over an order of magnitude higher than many metals. Generation efficiency should increase if the light-absorbing film is made with a large $\alpha$ material such as PDMS rather than the metallic films used in the past. ${ }^{3,6-8}$ Since PDMS is a transparent silicone, a suitable dye or pigment must be added to make it optically absorbing.

We spin coated and cured a mixture of PDMS, carbon black, ${ }^{9}$ and toluene onto a microscope glass slide. The film thickness is estimated to be $25 \mu \mathrm{m}$. The glass slide was then mounted on the bottom of a water tank (Fig. 1). A $5 \mathrm{~cm}$ focal length planoconvex lens focuses the laser pulse through the glass slide onto the black PDMS film. The excitation source is a directly modulated, single transverse mode InGaAsP diode laser $(\lambda=1.55 \mu \mathrm{m})$ followed by an erbium-doped fiber amplifier (EDFA). The EDFA consists of $10 \mathrm{~m}$ of erbiumdoped fiber pumped at $0.98 \mu \mathrm{m}$ using a fiber coupled InGaAs diode laser. A $10 \mathrm{~ns}$ laser pulse with an energy of $30 \mathrm{~nJ}$ was used for all studies. Ultrasound was detected by a spherically focused, $f / 1.4$ PVDF transducer with a focal length of $4.1 \mathrm{~mm}$. The transducer has a center frequency of

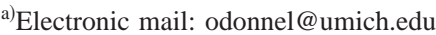

$45 \mathrm{MHz}$. The detected signal was amplified by $30 \mathrm{~dB}$ before data capture.

Figure 2(a) shows single shot acquisition of the detected signal. Accounting for the limited dynamic range of the digitizer, the signal to noise ratio (SNR) is measured to be $38 \mathrm{~dB}$. In comparison, Fig. 2(b) shows the signal generated from a $0.15-\mu \mathrm{m}$-thick chromium film deposited on a glass slide. The signal required an additional $30 \mathrm{~dB}$ of amplification before data capture. The SNR in this case is only $14 \mathrm{~dB}$. The thermoelastic effect in the black PDMS film therefore has an increased efficiency of $24 \mathrm{~dB}$ compared to the chromium film.

The spectrum of the PDMS signal, averaged 100 times, is shown by the solid curve in Fig. 3. The large fractional bandwidth is consistent with the tight time domain pulse. A crude model of the signal spectrum, shown by the dashed curve in Fig. 3, consists of multiplying the spectrum of the laser pulse with the square root of the pulse echo response of the transducer. Agreement is fairly good, indicating that the spectrum of the radiated acoustic pulse is comparable to the spectrum of the laser pulse.

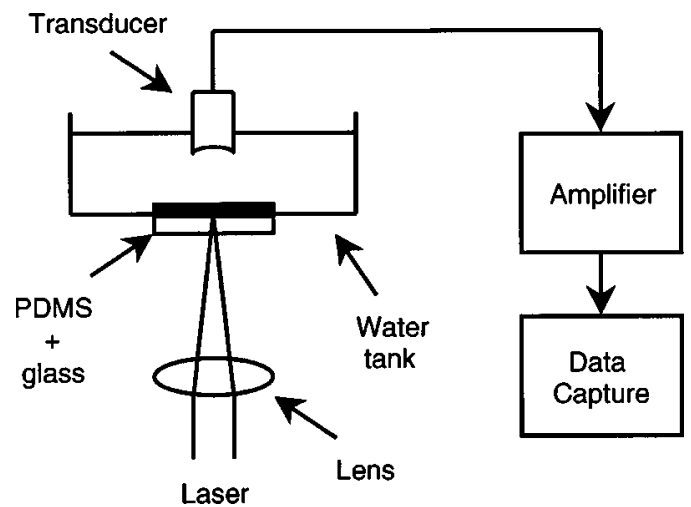

FIG. 1. Block diagram of experiment for thermoelastic generation of ultrasound. 


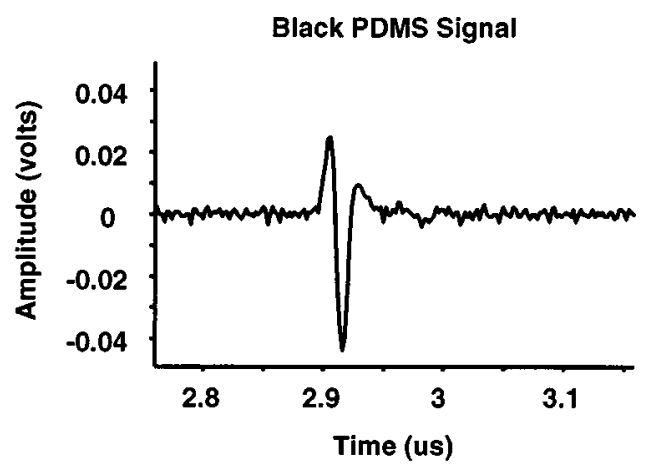

(a)

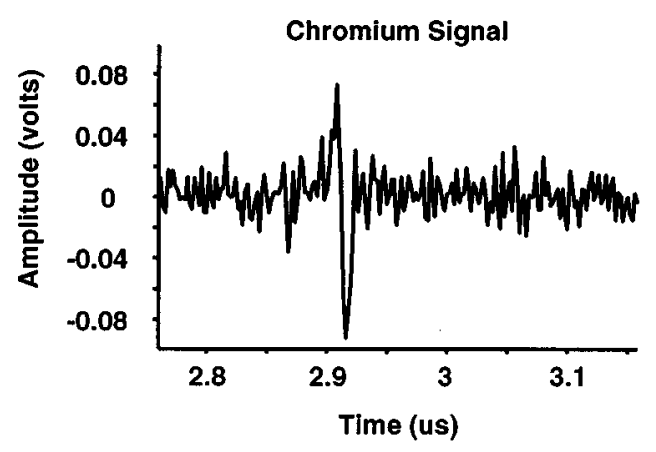

(b)

FIG. 2. Single shot acquisition of signal from (a) black PDMS and (b) chromium.

The effective size of the thermoelastic array element was estimated from the radiation pattern of the acoustic field. The radiation pattern from the black PDMS film was measured by scanning the transducer along an arc, covering an angular range of $\pm 45^{\circ}$ at $5^{\circ}$ intervals. All signals were averaged 100 times during data capture. The square markers in Fig. 4 show the measured radiation patterns for $15,35,50$, and $70 \mathrm{MHz}$, where the magnitude of the fast Fourier transform was used at each position to estimate the signal strength. Considerable energy is radiated at $+/-45^{\circ}$, even at 50 and $70 \mathrm{MHz}$. A fairly good fit to the data was produced with the first Rayleigh-Sommerfeld diffraction integral in the far field regime. ${ }^{10}$

$$
p(R, \theta) \propto \frac{\cos \theta}{R} \mathcal{B}\left[p\left(r_{0}, 0\right)\right]_{\rho=\sin \theta / \lambda},
$$

where $R=\sqrt{x^{2}+y^{2}+z^{2}}, \cos \theta$ is the obliquity factor, $p\left(r_{0}, 0\right)$ is the pressure specified on the plane $z=0$, and $\mathcal{B}[]$ repre-

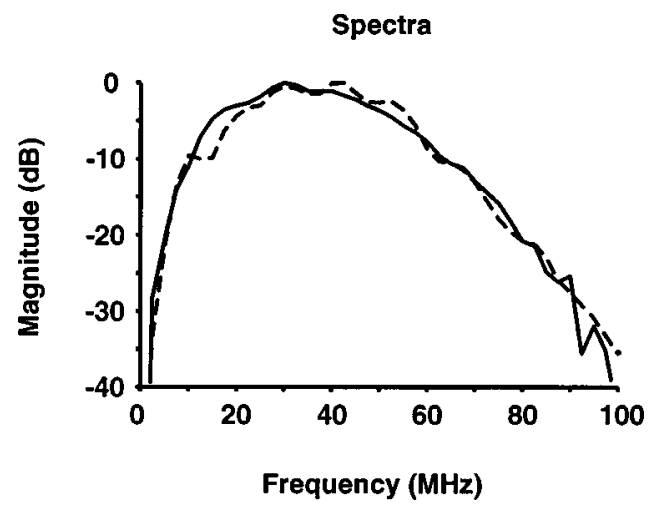

FIG. 3. Spectrum of signal (solid curve) and simulation (dashed curve).
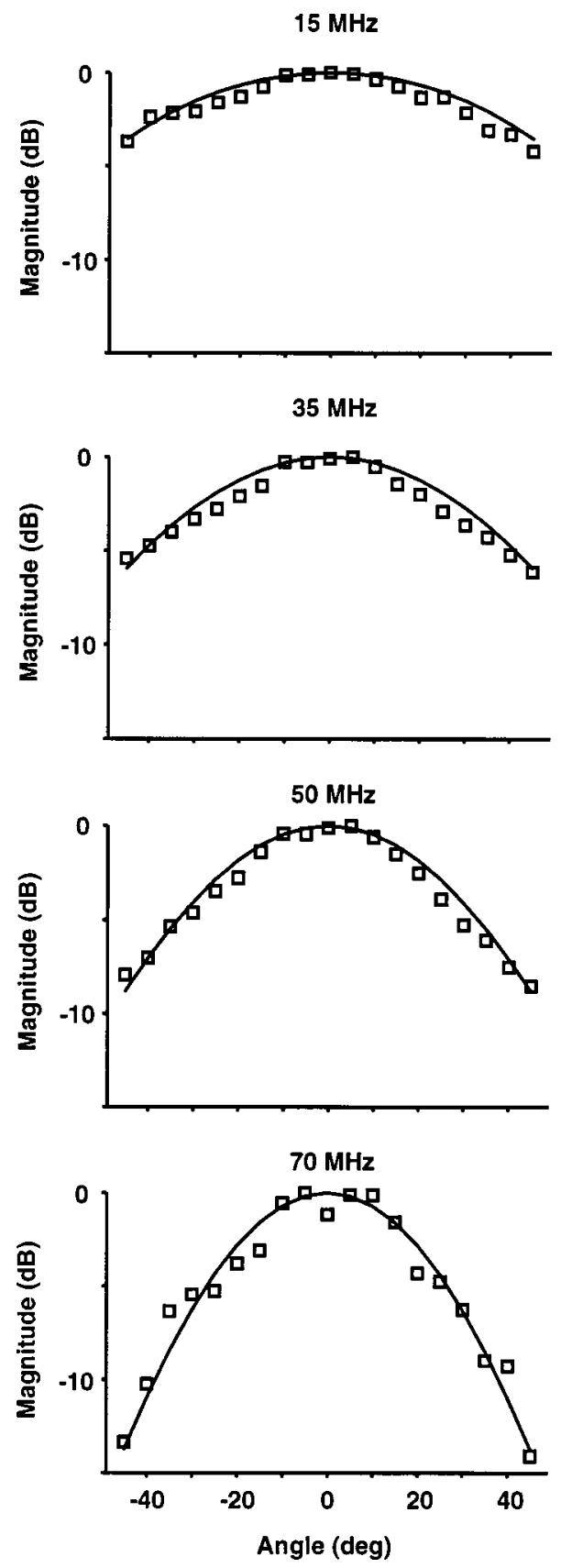

FIG. 4. Measured (square markers) and simulated (solid curve) radiation patterns at $15,35,50$, and $70 \mathrm{MHz}$.

sents the Fourier-Bessel transform operation. The angular integration performed by the transducer was also taken into account. The solid curves in Fig. 4 correspond to a Gaussian shaped circular aperture function for $p\left(r_{0}, 0\right)$ with a $1 / e^{2}$ diameter of $35 \mu \mathrm{m}$. The acoustic spot size appears to be considerably larger than the focus of the laser, estimated to be 15 $\mu \mathrm{m}$. A possible explanation for the larger than expected element size is optical scattering within the carbon black producing a halo surrounding the optical focus. Another possibility is that interfacial waves, excited along the PDMS-glass boundary, radiate longitudinal waves into the water as they propagate away from the optical focus.

The diffraction integral in Eq. (1) assumes a pressure release baffle surrounding the array element. We find it surprising that the model closely agrees with the data, considering that the glass substrate instead would seem to act more 
like a rigid baffle. Furthermore, any complex angular dependencies of the radiation field due to acoustic boundary conditions at the PDMS-glass and PDMS-water interfaces were smeared out by the angular integration performed by the detecting transducer. Radiation pattern measurements with finer spatial sampling are therefore required to gain a better understanding of the thermoelastic generation process.

We have demonstrated a conversion efficiency increase of $24 \mathrm{~dB}$ simply by using a light-absorbing film with a high thermal expansion coefficient. Further imaging improvement can be achieved by increasing the laser energy. We estimate the damage threshold of the PDMS film to be over an order of magnitude higher than current operating conditions. The temporal response of the black PDMS film appears to be short enough to generate ultrasound with bandwidths of 100 MHz. Radiation pattern measurements suggest that array element size is considerably larger than expected, but wide acceptance angles are still available for imaging applications at frequencies greater than $50 \mathrm{MHz}$. We note that in contrast to a piezoelectric array, the element spacing for a thermoelastic array can be separately controlled from the element size. Element spacing is limited by the resolution of the scanning system, regardless of the size of the element itself. Consequently, high quality electronic images at ultrasound frequencies greater than $50 \mathrm{MHz}$ are possible with a thermoelastic array.

Further work is required to reduce the effective size of the PDMS array element and design an accurate model of the generation process. We believe these results using an elastomeric film show the potential of the thermoelastic effect in producing phased arrays for high-frequency ultrasound imaging.

${ }^{1}$ R. M. White, J. Appl. Phys. 34, 3559 (1963).

${ }^{2}$ J. D. O'Keefe and C. H. Skeen, Appl. Phys. Lett. 21, 464 (1972).

${ }^{3}$ R. J. Von Gutfeld and H. F. Budd, Appl. Phys. Lett. 34, 617 (1979).

${ }^{4}$ J. A. Rogers, O. J. A. Schueller, and G. M. Whitesides, Appl. Phys. Lett. 72, 1951 (1998).

${ }^{5}$ Dow Corning Technical Data Sheet for Sylgard 184 silicone elastomer.

${ }^{6}$ M. J. Brienza and A. J. DeMaria, Appl. Phys. Lett. 11, 44 (1967).

${ }^{7}$ G. Cachier, J. Acoust. Soc. Am. 49, 974 (1971).

${ }^{8}$ H. Tanaka, K. Hattori, K. Sakai, and K. Takagi, Jpn. J. Appl. Phys., Part 2 36, L1415 (1997).

${ }^{9}$ Raven 2500 Ultra Carbon Black, Columbian Chemicals Company.

${ }^{10} \mathrm{~J}$. W. Goodman, Introduction to Fourier Optics, 2nd ed. (McGraw-Hill, New York, 1996), Chaps. 3 and 4. 